fact that these transformations do not reduce flow may mean that, as with the simplex algorithm for linear programming, rather a small number of different arrangements can be stepped through to reach the optimum.

It is too early to say whether these new ideas will lead to significantly greater efficiency in solving multicommodity problems. The paper deals only with certain specialized flow problems, so it is roughly analogous to the situation with the FordFulkerson approach before the out-of- kilter algorithm was developed. Lomonosov does not discuss algorithmic efficiency directly, nor does he give explicit examples, but it is likely that his methods will prove good for some kinds of problems. If this turns out to be the case, we may hope for extensions to more practically realistic problems and perhaps a large increase in the size of multicommodity problems that can be solved.

Alistair Mees is in the Department of Math ematics at the University of Western Australia, Nedlands, Western Australia 6009, Australia.

\title{
Molecular biology
}

\section{Self-incompatibility in plants}

\section{from John Fincham}

IN many families of flowering plants, exemplified by the Solanaceae (which includes the genera Nicotiana and Lycopersicon), self-pollination is prevented by a genetic system based on multiple alleles at what appears to be a single locus which is given the symbol $S$. Impressive progress towards the goal of discovering what the products of the $S$ allele are and how they relate to each other in molecular terms has recently been made by two groups, first by J. B. Nasrallah et al. from Cornell University (Nature 318, 263; 1985) and now by Anderson et al. of Melbourne University and reported elsewhere in this issue (Nature 321, 38; 1986).

It is known that pollination is blocked only when the same allele is present in the germinating pollen grain and the style down which it is trying to grow. Because wild populations of outbreeding species usually have tens or even hundreds of $S$ alleles, this system ensures that the great majority of random cross-pollinations are fertile. In some plant families, including the Cruciferae and its best-known genus Brassica, there is a variant system in which pollen expresses one or both of the $S$ alleles present in the diploid plant which bore it, even though each pollen grain, as a haploid product of meiosis, retains only one allele. The difference between the two systems is presumably one of timing of $S$-locus expression - post-meiotic in the Solanaceae and pre-meiotic in the Cruciferae - and does not necessarily imply any fundamental difference in the functions of the products of the respective $S$ loci. In each case, incompatibility evidently results from some kind of interaction between products of identical $S$ alleles.

What is the mechanism of this selfrecognition by $S$ alleles? There are two possibilities. If one supposes that the $S$ locus is really a single gene, encoding the same protein in pollen and style, one can think in terms of dimerization of similar protein monomers. located respectively on the cell surface of the pollen tube and in the cell sap of the style. The alternative is to postulate that the $S$ locus is a complex of at least two genes expressed respectively in pollen and style, with each $S$ allele encoding a stylar product and a different but complementary pollen product. The second model does not require the products of any particular $S$ allele (more accurately termed a haplotype) to bear any specific structural relationship to those of any other - each haplotype could evolve independently, subject to selection only for self-recognition. A bipartite structure of the $S$ locus has, in fact, long been postulated as an explanation of $S$ mutations, which tend to affect pollen and style reactivities independently.

The breakthrough by the Cornell group was the molecular cloning of a complementary (c)DNA sequence representing an abundant style-specific messenger (m)RNA in Brassica oleracea of genotype $S_{6} S_{b}$ (obtained by forced self-pollination). When used to probe restriction-endonuclease digests of DNA from plants carrying other $S$ alleles, this cDNA clone revealed a restriction fragment size polymorphism that was closely and perhaps inseparably linked to the $S$ locus; each $S$ allele was associated with its own specific array of restriction sites within the region covered by the probe. Moreover the cDNA, when cloned into an expression vector, was translated in Escherichia coli into a protein product that reacted with an antiserum made against a glycoprotein of relative molecular mass $\left(M_{\mathrm{r}}\right) \sim 85,000$ that had been found specifically in styles of plants bearing allele $S_{6}$. Only a partial sequence of the cDNA clone has been published so far.

The starting point for the Australian group was the finding of an abundant glycoprotein of $M_{\mathrm{r}} \sim 32,000$ in styles of Nicotiana alata (tobacco) plants carrying allele $S$. Somewhat surprisingly, no glycoprotein of comparable abundance was isolated by the same method from plants carrying other $S$ alleles, which raises the possibility that different $S$ alleles have very different stylar products. Determination of part of the $\mathrm{N}$-terminal amino-acid sequence of the $M_{\mathrm{r}} 32,000$ protein permitted the synthesis of a mixed DNA probe for the encoding gene, and the gene was indeed recovered by this means from a cDNA library made from mRNA of styles carrying $S_{2}$. Sequencing of the cDNA clone revealed an open reading frame encoding a 204-residue polypeptide chain with a 22-residue $\mathrm{N}$-terminal hydrophobic portion (presumed signal sequence) leading into the sequence already found at the N-terminus of the mature glycoprotein. The cDNA hybridized strongly to DNA of plants carrying $S_{2}$ and much more weakly to DNA of plants with other $S$ alleles, suggesting again that the different $S$ alleles have very subtantially different coding sequences and only remotely related stylar products. There is as yet no direct evidence about the extent and nature of the inter-allele differences within $N$. alata, but Anderson et al. do present some preliminary information from another Solanaceous species, the wild tomato Lycopersicon peruvianum. A part of the $\mathrm{N}$-terminal sequence has been obtained from two different stylar glycoproteins associated with different Lycopersicon $S$ alleles; these are clearly homologous with each other (although there are also several differences) and also with the $\mathrm{N}$-terminal sequence of the $N$. alata $\mathrm{S}_{6}$ protein. Disappointingly, but not surprisingly, no similarity is visible as yet between the Nicotiana or Lycopersicon sequences and the limited amount of sequence available from the Brassica $\mathrm{S}_{2}$ protein.

Clearly we are at the beginning of what is bound to be a period of intense activity as these highly promising observations are carried further. Several questions are now readily answerable. We should soon see some full-length comparisons between the DNA sequences of different $S$ alleles (or at least those parts of them governing the stylar products); it should soon become apparent if there is any homology between the Nicotiana/Lycopersicon and Brassica $S$ loci. More difficult, but probably not long delayed, will be the identification of $S$-allele products in the pollen tube and the cloning of the corresponding DNA sequences. If the $S$ locus really does have separate pollen- and style-specific products with seperate coding sequences, much may depend on how close together these sequences are in the genomic DNA. If they are separated by only tens of kilobases, walking from one to the other should not be difficult; but if they are hundreds of kilobases apart, as they could be and still be almost inseparably linked, there will be more of a problem.

John Fincham is Professor of Genetics at the University of Cambridge, Downing Street, Cambridge CB2 $3 E H, U K$. 